\title{
Can Vacancies Lubricate Dislocation Motion in Aluminum?
}

\section{Citation}

Lu, Gang, and Efthimios Kaxiras. 2002. "Can Vacancies Lubricate Dislocation Motion in Aluminum?" Physical Review Letters 89 (10). https://doi.org/10.1103/physrevlett.89.105501.

\section{Permanent link}

http://nrs.harvard.edu/urn-3:HUL.InstRepos:41384114

\section{Terms of Use}

This article was downloaded from Harvard University's DASH repository, and is made available under the terms and conditions applicable to Other Posted Material, as set forth at http:// nrs.harvard.edu/urn-3:HUL.InstRepos:dash.current.terms-of-use\#LAA

\section{Share Your Story}

The Harvard community has made this article openly available.

Please share how this access benefits you. Submit a story.

\section{Accessibility}




\title{
Can vacancies lubricate dislocation motion in aluminum?
}

\author{
Gang $\mathrm{Lu}$ and Efthimios Kaxiras \\ Department of Physics and Division of Engineering and Applied Science, \\ Harvard University, Cambridge, MA 02138
}

\begin{abstract}
The interaction of vacancy with dislocations in $\mathrm{Al}$ is studied using the Semidiscrete Variational Peierls-Nabarro model with $a b$ initio determined $\gamma$-surface. For the first time, we confirm theoretically the so-called vacancy lubrication effect on dislocation motion in $\mathrm{Al}$, a discovery that can settle a long-standing controversy in dislocation theory for fcc metals. We provide insights on the lubrication effect by exploring the connection between dislocation mobility and its core width. We predict an increased dislocation splitting in the presence of vacancy. We find that on average there is a weak repulsion between vacancies and dislocations which is independent of dislocation character.
\end{abstract}

Defects and their mutual interactions dominate the properties of materials that host them. Vacancies as point defects, have long been known to strongly interact with dislocations (line defects), and the study of their interactions represents one of most challenging problems in material science and engineering [1]. More than a decade ago, Benoit et al. discovered an interesting phenomenon in ultra-high-purity aluminum deformed at low temperature of $4.2 \mathrm{~K}$. They observed a marked decrease of elastic modulus in cold-worked $\mathrm{Al}$, which they attributed to vacancy enhanced dislocation mobility in $\mathrm{Al}$, a novel phenomenon they termed dislocation lubrication effect [2, 3]. Corroborative experimental evidence led these authors to conclude that vacancies, generated from cold-work or irradiation, are solely responsible for the enhanced dislocation mobility. This indeed is a quite intriguing result because traditionally vacancies are thought to lock dislocation motion by forming atmospheres around the dislocation 狛. Furthermore the lubrication effect may hold the key to resolve the long-standing controversy for Peierls stress $\left(\sigma_{p}\right)$ estimated from plastic deformation and from internal friction measurements. It is generally believed that kink pair formation (KPF) of dislocations is responsible for the Bordoni peaks observed in internal friction measurements of fcc metals [5, 6]. However $\sigma_{p}$ derived from KPF mechanism is in the order of $10^{-3} \mu$ ( $\mu$ is the shear modulus) far greater than the critical resolved shear stress (CRSS) estimated from plastic deformation experiments, which is around $10^{-5}$ to $10^{-4} \mu$ [7]. This has been regarded as a serious problem because it casts doubt on the well accepted KPF theory for the Bordoni peak in fcc metals. The controversy is particularly troublesome in light of the good agreement found in bcc and ionic crystals regarding $\sigma_{p}$ measured from internal friction and low temperature CRSS experiments [6]. Therefore the vacancy lubrication effect may settle the controversy by proposing that vacancies strongly interact with dislocations and as a consequence lower their $\sigma_{p}$ to the level that is consistent with the low temperature CRSS experiments and thus bridge the gap in $\sigma_{p}$ [2, 3. Finally if the lubrication mechanism turns out to be general, it may lead to an innovation in molding technology for materials with high $\sigma_{p}$ by introducing vacancies to the materials [8]. However interesting the lubrication effect may seem to be, it has not been widely accepted, which in our opinion, is due to poor understanding of the phenomenon. In fact, there is no complete theoretical work ever published to address this problem, to the best of our knowledge. Therefore it is the purpose of this paper to present the first ab initio study of the problem. As we will show in the following, our calculations not only provide theoretical support for the lubrication effect, they also reveal other important difference in dislocation properties that are associated with the presence of vacancies.

In this paper, we employed the recently developed Semidiscrete Variational Peierls-Nabarro (SVPN) model [9. 10] in conjunction with ab initio determined $\gamma$-surfaces 11. The SVPN model provides an ideal framework for multiscale simulations of dislocation properties, and it combines an atomistic ( $a b$ initio) treatment of the interactions across the slip plane and an elastic treatment of the continua on either side of the slip plane. The model has been shown to be quite successful in predicting dislocation properties by comparing its predictions against the direct atomistic simulations results 9 , 10]. For example, by using the $\gamma$-surface calculated from Embedded Atom Method (EAM), we obtained $\sigma_{p}$ for dislocations in $\mathrm{Al}$ that are in excellent agreement with that from direct atomistic simulations employing the same EAM potential [10]. More remarkably, a good agreement is also achieved for dislocations in $\mathrm{Si}[9]$ where the classic Peierls-Nabarro model fails owing to the its insufficiency to deal with narrow dislocations, such as dislocations in Si. Since one has no a priori knowledge regarding the size of dislocations in the presence of vacancies, the SVPN model seems to be particularly useful to explore the interaction of vacancy with dislocations. Besides $\sigma_{p}$, the model can also provide reliable results for other dislocation properties, such as partial separation distance and core width [10], as they are compared to direct atomistic simulations 13, 14. Thus the strength of this approach, when combined with ab initio calculations for $\gamma$-surface is that it produces essentially an atomistic simulation for dis- 
location properties without suffering from uncertainties associated with empirical potentials.

In the SVPN approach, the equilibrium structure of a dislocation is obtained by minimizing the dislocation energy functional [9, 10]

$$
U_{\text {disl }}=U_{\text {elastic }}+U_{\text {misfit }}+U_{\text {stress }}+K b^{2} \ln L,
$$

where

$$
\begin{gathered}
U_{\text {elastic }}=\sum_{i, j} \frac{1}{2} \chi_{i j}\left[K_{e}\left(\rho_{i}^{(1)} \rho_{j}^{(1)}+\rho_{i}^{(2)} \rho_{j}^{(2)}\right)+K_{s} \rho_{i}^{(3)} \rho_{j}^{(3)}\right] \\
U_{\text {misfit }}=\sum_{i} \Delta x \gamma\left(\vec{f}_{i}\right), \\
U_{\text {stress }}=-\sum_{i, l} \frac{x_{i}^{2}-x_{i-1}^{2}}{2} \rho_{i}^{(l)} \tau^{(l)}
\end{gathered}
$$

with respect to the dislocation Burgers vector density $\rho_{i}$. Here, $\rho_{i}^{(1)}, \rho_{i}^{(2)}$ and $\rho_{i}^{(3)}$ are the edge, vertical and screw components of the general Burgers vector density defined at the $i$ th nodal point as $\rho_{i}=\left(f_{i}-f_{i-1}\right) /\left(x_{i}-x_{i-1}\right)$, where $f_{i}$ and $x_{i}$ are the displacement vector and the coordinate of the $i$ th nodal point (atomic row). $\gamma\left(\vec{f}_{i}\right)$ is the $\gamma$-surface that is determined from ab initio calculations. $\tau^{(l)}$ is the external stress component interacting with the corresponding Burgers vector density $\rho^{(l)} \cdot \chi_{i j}$ is the discretized elastic energy kernel, and $K, K_{e}$ and $K_{s}$ are the pre-logarithmic elastic energy factors [9, 10]. The quantity $L$ entering the last term is the outer cutoff radius for the configuration-independent part of the elastic energy [12]. We identify the dislocation configuration-dependent part of the elastic energy and the misfit energy as core energy, i.e., $U_{\text {core }}=U_{\text {elastic }}+U_{\text {misfit }}$. The response of a dislocation to an external stress is achieved by minimization of the energy functional at the given value of the applied stress. An instability is reached when an optimal solution for the Burgers vector density distribution no longer exists, which is manifested numerically by the failure of the minimization procedure to convergence. $\sigma_{p}$ is then identified as the critical value of the applied stress giving rise to this instability.

In order to examine how vacancies change dislocation core structure by modifying atomic bonding across the slip plane, we carry out ab inito calculations for the $\gamma$ surface of Al with vacancies at the slip plane. Specifically, we select a supercell containing six Al layers in [111] direction with four atoms per layer, and remove one $\mathrm{Al}$ atom from the top layer (right below the designated slip plane) of the supercell to simulate a vacancy concentration at 4 at.\%. We should emphasize that 4 at.\% represents the vacancy concentration at the dislocation core region that we are interested, therefore it is much greater than the average vacancy concentration of the bulk material. The ab initio calculations are based on the pseudopotential plane-wave method with local density approximation 15] to the exchange-correlation functional [16]. A kinetic energy cutoff of $12 \mathrm{Ry}$ for the planewave basis is used and a $k$-point mesh consisting of $(8,8,4)$ divisions along the reciprocal lattice vectors is sampled for the Brillouin zone integration. Atomic relaxation is performed before we initiate the sliding. During the sliding process, atoms are allowed to move only along [111] direction while the atoms at the innermost two layers are held fixed. Volume relaxation is also performed for each sliding distance to minimize the tensile stress on the supercell.

The $a b$ initio determined $\gamma$-surface for $\mathrm{Al}$ with and without vacancies is presented in Fig. 1. In order to highlight the vacancy effect on $\gamma$-surface, we also summarize in Table I some important stacking fault energies for both $\mathrm{Al}$ and $\mathrm{Al}+\mathrm{V}$ (Al with vacancies) systems. These special stacking faults correspond to the various extremes along [121] ] and [101] directions of the $\gamma$-surface. It is clear that the vacancy lowers the intrinsic and un-

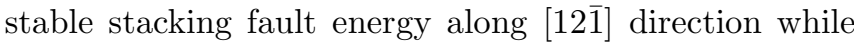
increases the run-on stacking fault energy and unstable stacking fault energy along [101] direction. Therefore it is not immediately clear how dislocation core structure can be changed by vacancies, and a detailed analysis based on SVPN model is needed. Since the experiments [2] have concluded that the change in elastic constants due to vacancies is not responsible for the observed lubrication effect, we will simply use the experimental elastic constants of pure $\mathrm{Al}$ in our calculations for $\mathrm{Al}+\mathrm{V}$ system [10].

Having determined all the necessary parameters entering the model, we can study the interaction of vacancy and dislocations using the SVPN model. We select four, namely, screw $\left(0^{\circ}\right), 30^{\circ}, 60^{\circ}$ and edge $\left(90^{\circ}\right)$ dislocations, all with the same Burgers vector, but different orientations, in our calculations. $\sigma_{p}$ calculated from the SVPN model for the dislocations with and without vacancies is listed in Table II. $\sigma_{p}$ represents the intrinsic mobility of a straight dislocation, and it relates to the kink pair formation energy, $2 W_{k}$, by

$$
2 W_{k}=\frac{\left(16 \mu b^{3} a^{3} \sigma_{p}\right)^{1 / 2}}{\pi},
$$

where $b$ is the Burgers vector and $a$ the distance between neighboring Peierls valleys [5, 6, 17]. The corresponding values for $\mathrm{Al}$ are $\mu=28.8 \mathrm{GPa}, b=2.85 \AA$ and $a=2.47 \AA$. By measuring $2 W_{k}$ (the activation energy for the Bordoni peak) in internal friction experiments, one can derive $\sigma_{p}$ according to Eq. (5) for the relevant dislocation. For example, using the experimentally measured value of $2 W_{k}(0.21 \mathrm{eV})$ for a screw dislocation, one obtains $\sigma_{p}=8 \times 10^{-3} \mu(224 \mathrm{MPa})$ for the screw dislocation in pure $\mathrm{Al}$ [6, 17]. This value of $\sigma_{p}$ is in excellent agreement with our model result, $8.82 \times 10^{-3} \mu$ for the same 
dislocation (Table II). Furthermore, the less definite measurement for the subsidiary peak (B1 peak) yields an activation energy ranging from 0.12 to $0.16 \mathrm{eV}$, which corresponds to $\sigma_{p}$ in the range of 2.8 to $4.6 \times 10^{-3} \mu$ (80 to 130 $\mathrm{MPa}$ ) for the $60^{\circ}$ dislocation. This value also agrees well with our result for the same dislocation $\left(3.40 \times 10^{-3} \mu\right)$. The overall consistency between the theoretical and experimental values for $\sigma_{p}$ indicates the reliability of our model and establishes the basis for further study of vacancy effect.

When vacancies are introduced at the slip plane but are not adsorbed by a dislocation line, we find that $\sigma_{p}$ for various dislocation is lowered by more than one order of magnitude (except for the edge dislocation), as shown in Table II. Therefore we have confirmed the vacancy lubrication effect theoretically for the first time since its proposal. The fact that this lubrication effect is observed for various dislocations suggests a generic nature of the underlying mechanism. In order to shed light on this general mechanism, we have calculated dislocation core width which is defined as the atomic spacing over which the relative displacement of the dislocation changes from $1 / 4 b$ to $3 / 4 b$ [10]. It is generally believed that $\sigma_{p}$ is exponentially lowered with the increase of dislocation half-width according to the Peierls-Nabarro model [10, 12]. The calculated dislocation half core width $(\zeta)$ is presented in Table II. It is found that in the presence of vacancy, dislocation becomes $60 \%$ to $90 \%$ wider, which we believe is due to the reduced slope of the $\gamma$ -

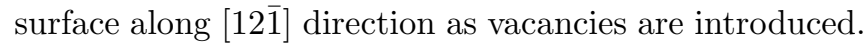
Since lattice restoring force, represented by the slope of $\gamma$-surface, is weakened by the vacancies, the repulsive elastic force resulting from the continuous distribution of infinitesimal dislocations dominates, leading to a wider dislocation core and therefore enhanced dislocation mobility. Although the vacancy lubrication effect may be qualitatively understood from above argument by a careful inspection of the $\gamma$-surface, one has to resort to SVPN model to obtain reliable values of $\sigma_{p}$ in order to make a quantitative comparison. As shown in Table II, vacancies can bring $\sigma_{p}$ down to the values derived from the plastic deformation experiments $\left(10^{-5}\right.$ to $\left.10^{-4} \mu\right)$, therefore bridge the gap for $\sigma_{p}$ between the internal friction measurement and the measurement of CRSS at low temperature.

In order to gain more insights into the interaction of vacancy and dislocations, we have calculated dislocation Burgers vector density for pure $\mathrm{Al}$ and $\mathrm{Al}+\mathrm{V}$, shown in Fig. 2. It is found that dislocations tend to dissociate more into partials in the presence of vacancy. This behavior is obviously associated with the fact that the intrinsic stacking fault energy is reduced by vacancy. The result cautions us to be more careful in interpreting TEM data for partial separation distances because accidentally introduced vacancies could change the result significantly. We have also calculated binding energies of vacancies to dislocation cores, summarized in Table II. The binding energy is defined as difference between dislocation core energy with and without the presence of vacancy. Overall we find that the binding energies are not sensitive to dislocation character, and more importantly they are all marginally positive. The positive binding energy indicates that dislocation is energetically less stable with vacancies nearby. Our result for positive value of binding energy qualitatively agrees with the findings from atomistic simulations carried out for $\mathrm{Cu}$ [1]. The small value of binding energy we found in $\mathrm{Al}$ may relate to the fact that the dislocations are dissociated into partials in $\mathrm{Al}$. It is believed that the vacancy binding energy to dissociated dislocations is considerably lower than that to the same dislocations in the non-dissociated condition [1]. Of course one has to bear in mind that the binding energy we obtained represents an average value over all possible sites for a vacancy at the core of the dislocation.

To conclude, we have studied the interaction of vacancy with dislocations in $\mathrm{Al}$ using the SVPN model with $a b$ initio determined $\gamma$-surface. We confirm the experimental finding of vacancy lubrication effect in $\mathrm{Al}$. We propose that vacancies can weaken the lattice restoring force across the slip plane, which leads to a wider dislocation spreading, and thus higher dislocation mobility. We find that $\sigma_{p}$ of the dislocations is lowered by more than one order of magnitude in the presence of vacancy, which bridges the gap between $\sigma_{p}$ observed from different experiments, resolving one of the long-standing problems in dislocation theory. This work represents the first theoretical effort to challenge the traditional point of view that regards vacancy as a locking agent for dislocation motion. We predict that vacancies can increase partial separation distance in $\mathrm{Al}$, and finally we find there exists a weak repulsion between dislocations and vacancy, which is independent of the dislocation character.

We acknowledge the support from Grant No. F4962099-1-0272 through the U.S. Air Force Office for Scientific Research.

[1] R.W. Balluffi and A.V. Granato, in Dislocations in Solids, edited by F.R.N. Nabarro (North-Holland, Amsterdam, 1979), Vol. 4, p.1.

[2] J. Lauzier, J. Hillairet, A. Vieux-Champagne, and W. Benoit, J. Phys. Condens. Matter, 1, 9273 (1989); J. Lauzier, J. Hillairet, G. Gremaud and W. Benoit, ibid, 2, 9247 (1990).

[3] W. Benoit, G. Gremaud and B. Quenet, Mater. Sci. Eng. A 164, 42 (1993).

[4] F.R.N. Nabarro, Theory of Crystal Dislocations, (Dover, New York, 1987).

[5] A. Seeger, Philos. Mag. 1, 651 (1951).

[6] G. Fantozzi, C. Esnouf, W. Benoit and G. Ritchie, Prog. Mater. Sci, 27, 311 (1982).

[7] A. Seeger and P. Schiller, in Physical Acoustics, edited 
by W. Mason (Academic Press, New York, 1966), Vol. 3, p.366.

[8] T. Kosugi and T. Kino, Mater. Sci. Eng. A 164, 368 (1993).

[9] V. V. Bulatov and E. Kaxiras, Phys. Rev. Lett. 78, 4221 (1997).

[10] G. Lu, N. Kioussis, V. V. Bulatov, and E. Kaxiras, Phys. Rev. B 62, 3099 (2000), Philos. Mag. Lett. 80, 675 (2000).

[11] When a crystal is cut along its slip plane and the upper half is displaced relative to the lower by a vector $\vec{f}$, the energy increase per unit area is defined as $\gamma$ and the en- ergy surface $\gamma(\vec{f})$, with $\vec{f}$ spanning the entire slip plane, is called the $\gamma$-surface.

[12] J.P. Hirth and J. Lothe, Theory of Dislocations, 2nd ed. (Wiley, New York, 1992).

[13] V.V. Bulatov, O. Richmond, and M.V. Glazov, Acta Mater. 47, 3507 (1999).

[14] Q.F. Fang and R. Wang, Phys. Rev. B 62, 9317 (2000).

[15] W. Kohn and L. Sham, Phys. Rev. 140, A1133 (1965).

[16] J. Perdew and A. Zunger, Phys. Rev. B 23, 5048 (1984).

[17] W. Benoit, M. Bujard and G. Gremaud, Phys. Stat. Solidi. A104, 427 (1987). 

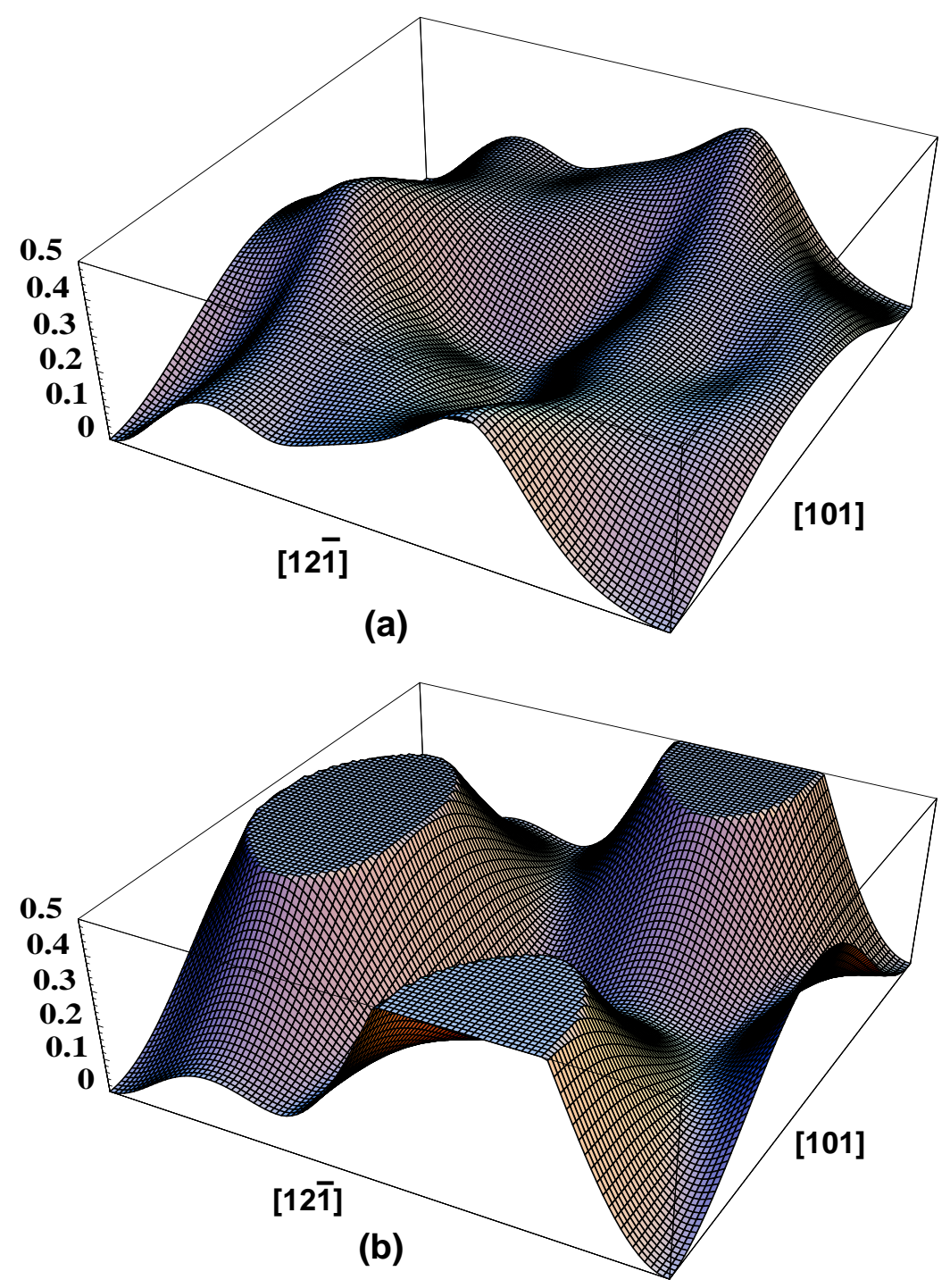

FIG. 1: The $\gamma$-surface $\left(\mathrm{J} / \mathrm{m}^{2}\right)$ for displacements along a (111) plane for (a) pure $\mathrm{Al}$ and (b) $\mathrm{Al}+\mathrm{V}$ systems. The corners of the plane and its center correspond to identical equilibrium configuration, i.e., the ideal lattice. The two surfaces are displayed in exactly the same perspective and on the same energy scale to facilitate comparison. The $\gamma$-surface of $\mathrm{Al}+\mathrm{V}$ is truncated to emphasize the more interesting region.

TABLE I: Fault vectors and energies $\left(\mathrm{J} / \mathrm{m}^{2}\right)$ for some important stacking faults of the pure $\mathrm{Al}$ and the $\mathrm{Al}+\mathrm{V}$ systems.

\begin{tabular}{lccc}
\hline \hline & Vector & $\mathrm{Al}$ & $\mathrm{Al}+\mathrm{H}$ \\
\hline Intrinsic stacking & $1 / 6[12 \overline{1}]$ & 0.164 & 0.105 \\
Unstable stacking & $1 / 10[12 \overline{1}]$ & 0.224 & 0.143 \\
Unstable stacking & $1 / 4[101]$ & 0.250 & 0.427 \\
Run-on stacking & $1 / 3[12 \overline{1}]$ & 0.400 & 0.831 \\
\hline \hline
\end{tabular}




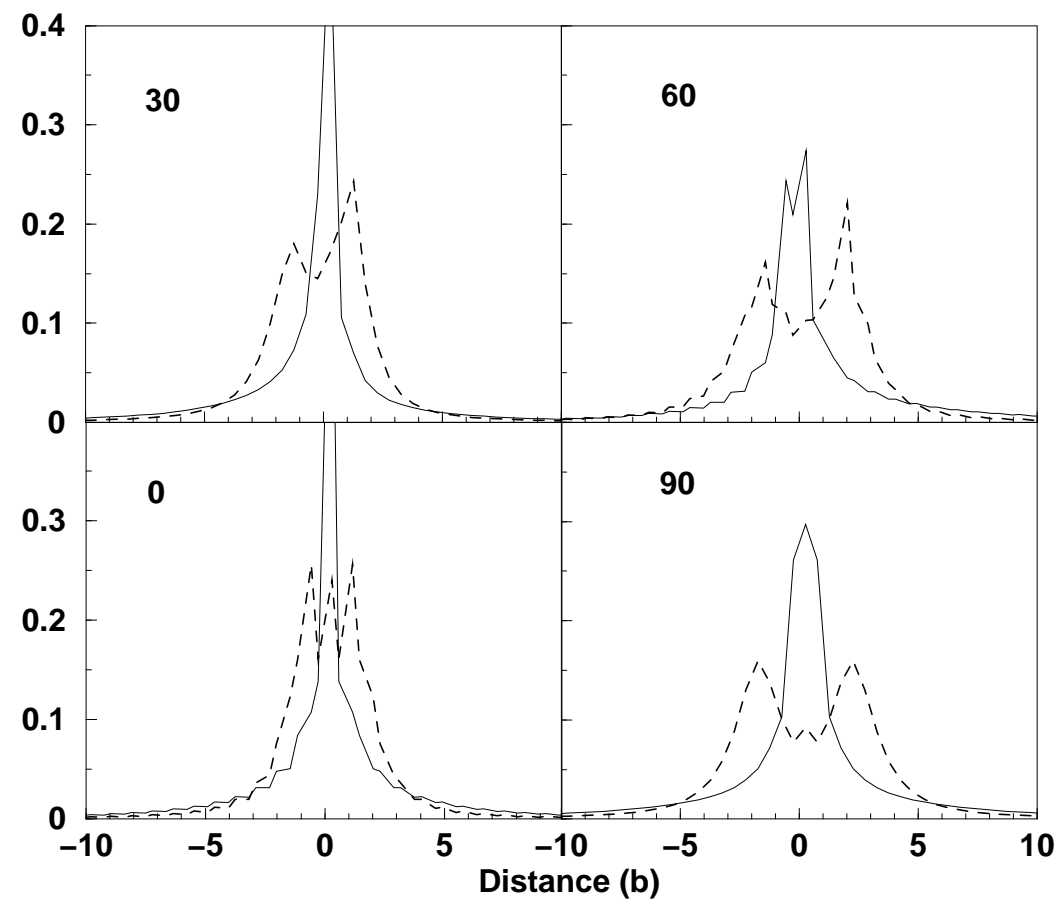

FIG. 2: Dislocation Burgers vector density for four dislocations (clockwise) : screw $\left(0^{\circ}\right), 30^{\circ}, 60^{\circ}$ and edge $\left(90^{\circ}\right)$ for the pure $\mathrm{Al}$ (solid lines) and the $\mathrm{Al}+\mathrm{V}$ (dashed lines) systems. The peaks in the density plot represent partial dislocations.

TABLE II: Peierls stress $\left(\sigma_{p}, 10^{-3} \mu\right)$, half core width $(\zeta$, $\AA)$, core energies $\left(U_{\text {core }}, \mathrm{eV} / \AA\right)$ for the four dislocations in the pure $\mathrm{Al}$ and the $\mathrm{Al}+\mathrm{V}$ systems and binding energy $\left(U_{b}\right.$, $\mathrm{eV} /$ vacancy) for the four dislocations.

\begin{tabular}{cccccc}
\hline \hline & & screw & $30^{\circ}$ & $60^{\circ}$ & edge \\
\hline$\sigma_{p}$ & $\mathrm{Al}$ & 8.82 & 1.77 & 3.40 & 0.10 \\
& $\mathrm{Al}+\mathrm{V}$ & 0.69 & 0.10 & 0.26 & 0.05 \\
\hline$\zeta$ & $\mathrm{Al}$ & 2.1 & 2.5 & 3.0 & 3.5 \\
& $\mathrm{Al}+\mathrm{V}$ & 3.2 & 4.0 & 5.6 & 6.3 \\
\hline$U_{\text {core }}$ & $\mathrm{Al}$ & -0.084 & -0.110 & -0.168 & -0.198 \\
& $\mathrm{Al}+\mathrm{V}$ & -0.046 & -0.073 & -0.133 & -0.164 \\
\hline$U_{b}$ & & 0.038 & 0.037 & 0.035 & 0.034 \\
\hline \hline
\end{tabular}

